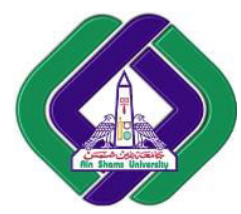

International Journal of Intelligent Computing

and Information Sciences

\title{
COMPARATIVE STUDY OF ROUTING PROTOCOLS FOR MOBILE AD HOC NETWORKS
}

Adel F. El-Kabbany,

Higher Technology Institute,

Kabany_2008@yahoo.com
Hanafy M. Ali,

Computers and Systems

Engineering Depart.,-Faculty of

Engineering, Minia University, El

Minia, Egypt

hmali@mu.edu.eg

\author{
Aziza I. Hussein ${ }^{1,2}$ \\ ${ }^{1}$ Computers and Systems Eng. Dept. \\ Minia University, Minia, Egypt \\ ${ }^{2}$ Electrical and Computer Eng. Dept. \\ Effat University, Jeddah, Saudi Arabia \\ azibrahim@effatuniversity.edu.sa
}

\author{
Dr. Ben. Tawfeek \\ Computers and Systems \\ Engineering Depart.,-Faculty \\ of Engineering, Suze Canal \\ University, Suze Canal, Egypt \\ benbellat@gmail.com
}

\begin{abstract}
Mobile Ad-hoc Network (MANET) is an infrastructure less and decentralized network which need a robust dynamic routing protocol. Many routing protocols for such networks have been proposed so far to find optimized routes from source to the destination and prominent among them are Dynamic Source Routing (DSR), Ad-hoc On-Demand Distance Vector (AODV), and DestinationSequenced Distance Vector (DSDV) routing protocols. The performance comparison of these protocols should be considered as the primary step towards the invention of a new routing protocol. This paper presents a performance comparison of proactive and reactive routing protocols DSDV, AODV and DSR based on QoS metrics (packet delivery ratio, average end-to-end delay, throughput, and jitter), normalized routing overhead and normalized MAC overhead by using the NS-2 simulator. In this work, the performance comparison is conducted by varying mobility speed, a number of nodes and data rate. The comparison results show that AODV performs optimally well not the best among all the studied protocols.
\end{abstract}

KEYWORDS: Mobile Ad hoc Network (MANET), AODV, DSDV, DSR, NS-2 simulator, and QoS metrics

\section{Introduction}

A Mobile Ad hoc Network (MANET) is a collection of mobile platforms that form a dynamic infrastructure-less communication network wherever it is required. The nodes in the MANET network acts as routers that discover and maintain routes to other nodes in the network. Quick and easy establishment of such networks makes them feasible to be used in military, disaster area recovery and in other environments where no infrastructure exists or it has been destroyed. Since mobile nodes move in various directions causing existing links to breaking and the establishment of new routes, routing in such networks is a challenging task. The mobility (i.e. how nodes move) of mobile nodes plays a significant role in the performance of routing protocols. Routes between two communicating nodes may consist of multiple hops through other nodes in the network. Therefore, finding and maintaining routes in MANET is nontrivial. Several routing protocols have been developed for mobile ad hoc networks. Such protocols must deal with typical limitations of these networks which include low bandwidth, high power consumption, and high error rates. Figure 1 shows the categorization of these routing protocols. In tabledriven routing protocols, all the nodes need to maintain the routing information in the tables and update it periodically even if they need to communicate or not. In this approach, pathfinding latency is small but 
the overhead is too high since a path which is not used for a long time is still maintained and updated. Examples include Destination-Sequenced Distance-Vector (DSDV) [1-10].

A mobile ad hoc network (MANET) is an autonomous, self-configuring network of mobile nodes that can be formed without the need of any pre-established infrastructure or centralized administration. MANETs are extremely flexible and each node is free to move independently, in any random direction. Each node in MANET maintains continuously the information required to properly route traffic. The three widely used routing protocols have been investigated and compared: Destination Sequenced Distance Vector (DSDV), Ad-hoc On-demand Distance Vector(AODV) and Dynamic Source Routing (DSR). MANETs are a kind of wireless ad-hoc network (WANET) that usually has a routable networking environment on top of a link layer ad hoc network. MANETs consist of a peer-to-peer, selfforming, self-healing network. Different protocols are then evaluated based on measures such as the packet drop rate, the overhead introduced by the routing protocol, end-to-end packet delays, network throughput, ability to scale, etc.[10-20].

the MANET routing protocols are classified into many routing protocols that proposed for mobile ad hoc networks such as classified under the following two criteria, proactive (Table -Driven) routing protocols and reactive (On-Demand) routing protocols. The Proactive Routing Protocols, in proactive routing protocols, nodes continuously map their circumstances nodes and spread this information periodically to all other nodes in the network. The proactive approach has the advantage that paths through the networks are already set up when nodes initiate traffic to a destination. This minimizes the delay in communication and allows nodes to quickly determine which nodes are reachable in the network.

Reactive routing protocols take the approach of only building a route when it needs it when nodes want to initiate traffic to a destination. The reactive protocol initiates a route by disseminating a route request into the network usually by flooding. The destination or an intermediary node with a route to the destination sends back a reply with the requested route information. Routing tables become soft routes and are removed when traffic is no longer sent and route discovery procedure then has to be repeated when traffic is needed to sent again. The destination or an intermediary node with a route to the destination sends back a reply with the requested route information. Routing tables become soft routes and are removed when traffic is no longer sent and route discovery procedure then has to be repeated when needed to sent again. On-demand term means routing creates routes only when needed by the source node An example of this type is dynamic source routing protocol (DSR) and ad hoc on demand distance vector protocol (AODV), When a node requires a route to a destination, it set up a route discovery process within the network [2-8,11-16,21,22].

\section{MANET Routing Protocols}

The most commonly adopted routing protocols have been chosen are DSDV, DSR, and AODV.

\subsection{DSDV Routing Protocol}

The destination sequenced distance vector (DSDV) routing protocol is a proactive routing protocol (table driven) based on classical Bellman-Ford routing algorithm [2-8].DSDV protocol drives the route by using route table basic, each node in the network maintains a routing table for the transmission of the packets. These nodes record for all the available destinations IP addresses, next hop IP address and the number of hops required to reach each destination in the routing table. The sequence number is used to distinguish routes from new ones and thus avoid the formation of loops. The data broadcast by each node will contain its new sequence number and the following information for each new route; the 
destination address, the number of hops required to reach the destination, and the new sequence number, originally generated by the destination. The mobile host cause broke links as they move from place to place within the network.

\subsection{DSR Routing Protocol}

The Dynamic Source Routing protocol (DSR) developed in 1998. It is a simple and efficient routing protocol designed specifically for multi-hop wireless ad hoc networks [11,19,23]. DSR is a reactive protocol that uses source routing rather than hop-by-hop routing, with each packet to be routed carrying in its header the complete, ordered list of nodes through which the packet must pass. The advantage of source routing is that intermediate nodes do not need to maintain updating routing information they forward the packets themselves already contain all the routing decisions. DSR protocol is consists of two mechanisms: route discovery and route maintenance. Route Discovery is the mechanism by which a source node wishing to send a packet to a destination node obtains a source route to the destination. Route Maintenance indicates that a source route is broken.

\subsection{AODV Routing Protocol}

AODV is an efficient routing protocol for MANET. It borrows most of the advantages basic from DSR and DSDV routing protocols. The on-demand route discovery and route maintenance from DSR and hop by hop routing, also the usage of node sequence numbers from DSDV bring into the algorithm very useful with topology and routing information [2,3,6,7,11-13,23].AODV is a reactive protocol. The routes are made only when they are needed. It uses routing tables, one entry per destination, and sequence numbers to figure out whether routing information is up to date and to stop routing loops. An important feature of AODV is the maintenance of time-based states in each node. A routing entry not recently used is expired. In case of a route is broken, the neighbors can be informed. Route discovery depends on query and reply cycles, and route information is saved in all intermediate nodes by the length of the route in the mold of route table entries. The following control packets are used:

1. Routing request message (RREQ) is transmitted by a node requiring a route to another node,

2. Routing reply message (RREP) is without a casing back to the source of RREQ, and route

3. Error message (RERR) is sent to inform other nodes of the loss of the link.

4. HELLO, messages are used for detecting and monitoring links to neighbors.

Each routing table entry contains the following data:

1. Destination address.

2. Next hop.

3. A number of hops.

4. Destination sequence number.

5. Active neighbors for this route.

Figure1(a): AODV Route Request (RREQ) Contents

\begin{tabular}{|l|l|l|l|l|l|l|}
\hline $\begin{array}{l}\text { Source } \\
\text { Address }\end{array}$ & Request ID & $\begin{array}{l}\text { Source } \\
\text { Sequence No }\end{array}$ & $\begin{array}{l}\text { Destination } \\
\text { Address }\end{array}$ & $\begin{array}{l}\text { Destination } \\
\text { Sequence No }\end{array}$ & Hop Count \\
To Live
\end{tabular}


Figure1(b): AODV Route Request (Route Reply RREP Contents)

\begin{tabular}{|l|l|l|l|}
\hline $\begin{array}{l}\text { Source } \\
\text { Address }\end{array}$ & $\begin{array}{l}\text { Destination } \\
\text { Address }\end{array}$ & $\begin{array}{l}\text { Destination } \\
\text { Sequence No }\end{array}$ & $\begin{array}{l}\text { Hop } \\
\text { Count }\end{array}$ \\
\hline
\end{tabular}

Figure 1: (a) AODV Route Request (RREQ) Contents,

(b) AODV Route Request (Route Reply RREP Contents)

\section{Simulation Environment and Parameters}

In our simulating, the mobile ad-hoc network (MANET) nodes are set of mobile nodes, a Source, and Destination. Each MANET node has a capability of transferring the packet from one end to other which doesn't have a fixed route because the network is Dynamic which indicates that all the mobile nodes in the network are moving [24-26]. There are different parameters on which our System is designed. Our MANET network is a wireless channel with radio propagation model and MAC type Mac/802_11 the communication of the network is done in the Link Layer of the OSI model. The protocol used for routing is AODV, DSR, and DSDV. We have used to agents UDP (user datagram protocol) and TCP (Transmission control protocol). The applications used are FTP (File transfer protocol) and CBR (Constant bitrate) because of this two application establishment of the connection within the network is done. The simulations were performed using Network Simulator 2 (NS-2.34), particularly popular in the ad hoc networking community [25].Simulation parameters used in our simulation model are shown in table 3 .

Table 1: Simulation Parameters

\begin{tabular}{|c|c|c|c|}
\hline Parameter & Value & Parameter & Value \\
\hline Simulator & NS- 2.34 & $\begin{array}{l}\text { Number of mobile } \\
\text { nodes }\end{array}$ & $20-100$ \\
\hline Channel type & $\begin{array}{l}\text { Channel/Wireless } \\
\text { channel }\end{array}$ & Source Type & $\begin{array}{lll}\text { CBR } & \text { (constant bit } \\
\text { rate) }\end{array}$ \\
\hline Mac Type & Mac / 802.11 & Simulation Time & $300 \mathrm{~s}$ \\
\hline $\begin{array}{l}\text { Network interface } \\
\text { type }\end{array}$ & Phy/ Wireless Phy & Routing Protocols & $\begin{array}{l}\text { DSDV, AODV, and } \\
\text { DSR }\end{array}$ \\
\hline $\begin{array}{l}\text { Radio - propagation } \\
\text { Type }\end{array}$ & $\begin{array}{l}\text { Propagation/ Two rays } \\
\text { round wave }\end{array}$ & Number of connection & 20 \\
\hline Network queue Type & Queue/ Drop Tail & Data Rate & 2-20 packet/ second \\
\hline Link Layer Type & $\mathrm{LL}$ & Pause time & 5 second \\
\hline Antenna & $\begin{array}{l}\text { Antenna/ } \\
\text { Antenna }\end{array}$ & Packet Size & 512 \\
\hline $\begin{array}{l}\text { Maximum packet in } \\
\text { ifq }\end{array}$ & 50 & Mobility Model & Random Waypoint \\
\hline Area $(\mathrm{M} * \mathrm{M})$ & $1000 * 1000$ & Transmission Range & $250 \mathrm{~m}$ \\
\hline Mobility Speed & $0-20 \mathrm{~m} / \mathrm{s}$ & & \\
\hline
\end{tabular}

Many papers proved that AODV and DSR have more advantages than DSDV in many performance metrics such as overhead, throughput, an end to end delay and packet delivered ratio (PDR). b. AODV 
has advantages than DSR and vice versa in some cases, for a performance comparison between those protocols.

\subsection{Evaluation Metrics}

Six metrics are used to evaluate our protocols, which are an end-to-end delay, Packet delivery ratio, Throughput, Jitter, normalized routing overhead, and Normalized MAC Overhead.

1) End-to-end delay is the average time delay for data packets to reach from the source node to the destination node. It includes processing, queuing and propagation delay of the link. The performance is better when packet end-to is low.

2) Packet delivery ratio (PDR) is the ratio between the number of packets transmitted by a traffic source and the number of packets received by a traffic sink; it represents the maximum throughput that the network can achieve. A high packet delivery ratio is desired in the study of performance metrics.

3) Throughput is the total packets successfully delivered to individual destinations over total time.

4) Jitter is the delay variation between each received data packets: The variation in the packet arrival time should be minimized to have better performance in Mobile Ad-hoc Networks.

5) Normalized Routing/Control Overhead Normalized Routing Overhead is defined as the number of routing packets "transmitted" per data packet "delivered" at the destination. The performance is better when routing overhead low.

6) Normalized MAC Overhead is defined as the fraction of all control packets (routing control packets, Clear-to-Send (CTS), Request-to-Send (RTS), Address Resolution Protocol (ARP), requests and replies, and MAC ACK total number of successfully received data packets.

\section{Results}

The simulation results are shown in the following section in the form of graphs. Graphs show a comparison between the three protocols on the basis of the above-mentioned metrics by varying mobility speed of the nodes, network size, and the load.

\subsection{Varying the node speed or dynamic property of the network in the first set of simulations:}

The mobility speed of the nodes is varied. The nodes start with a low velocity of $5 \mathrm{~m} / \mathrm{s}(18 \mathrm{~km} / \mathrm{h})$ and then the node velocity increases up to $20 \mathrm{~m} / \mathrm{s}(72 \mathrm{~km} / \mathrm{h})$. The data rate is kept constant at 10 packets/s $(40.960 \mathrm{kbps})$ and the no. of nodes and connections are fixed at 50 and 20 respectively. Packet delivery ratio in Fig. (2a) shows the packet delivery ratio of the protocols AODV, DSR, and DSDV. AODV and DSR almost show the similar performance. Packet delivery ratio for the protocols decreases as speed increases. This is because, at higher speeds, link breakage may occur more frequently and therefore a packet loss fraction is increased. Although the packet delivery fraction of all the protocols decreases as speed increases, DSDV's packet delivery fraction decreases in a more rapid fashion due to its excessive channel usage by regular routing table updates.

Furthermore, as mobility speed increases, more event-triggered updates are generated, resulting in even more packet delivery fraction decrease. This problem is not present in AODV, DSR since routes are generated only on-demand. Average end to end delay Fig.(2b) shows the average end to end delay of the three routing protocols. With the increase of movement speed, topology change may occur more frequently and thus the probability of broken links increases. Broken links may cause additional route recovery process and route discovery process. This leads to increase in average end-to-end delay of packets as the node speed increases. AODV protocol performs well for the dynamic network than DSR and DSDV on average end to end delay because it adopts both proactive and reactive features. In DSDV routing protocol, when the route break occurs in the network, the uplink and downlink nodes generate hello packets after waiting for the hello interval and the hello packets are propagated in the network with some delay (propagation delay). Moreover, some processing delay is also created due to the exchange and the updates of the routing tables at the nodes. Both these processing and the propagation delays are 
responsible for the increased end to end delay in case of DSDV.DSR end to end delay increases at high mobility speed because it suffers from stale route cache problem. Also, DSR is source path routing and when mobility is more in the network, less number of route replies is successfully received by the CBR sources. Throughput Again in the fig (2c), the throughput of AODV and DSR is more than DSDV because when the mobility speed of the node is increased, the throughput of DSDV decreases more as the node is busy in updating its routing table. DSR and AODV throughput is also decreased but its value is nominal. Jitter Figure (2d) shows the delay jitter of the three routing protocols. Jitter is dependent on delay as in jitter; variation of the packet arrival time is calculated in the network on the receiver side. That's why the jitter is almost in the same fashion for all the protocols like delay.

DSR has lower jitter on less speed because it utilizes the route information stored in the route cache for creating the connection but has more jitter variation above $10 \mathrm{~m} / \mathrm{s}$ because, at higher speeds, the DSR node cache information is stale for the other nodes. Normalized Routing Overhead Figure (2e) shows the normalized routing load. DSDV has the best performance with an increase of the routing load at a higher mobility.

AODV routing overhead is more than DSR and DSDV because it generates more no. of control packets to find a fresh enough route to the destination node. It increases when nodes move at higher speeds. DSR has higher routing load than DSDV due to its source path routing and stale route cache problem at increased mobility. But actually, the control overhead is measured based on the size of control packets in terms of bytes in the network. Size of control packets (in bytes) generated by DSDV and DSR protocols is greater than AODV protocol and when the size of the control packets increases, then the packets need to be fragmented, and it is difficult to handle fragmented packets in the mobile ad hoc network. That's why DSR is not suitable for big ad hoc network. From this observation, it can be concluded that AODV protocol is best suitable for the dynamic network. Normalized MAC overhead Figure (2f) shows the normalized MAC overhead. AODV has higher normalized MAC load than DSR. DSDV is the most stable protocol in terms of the normalized MAC load in networks with varying mobility.

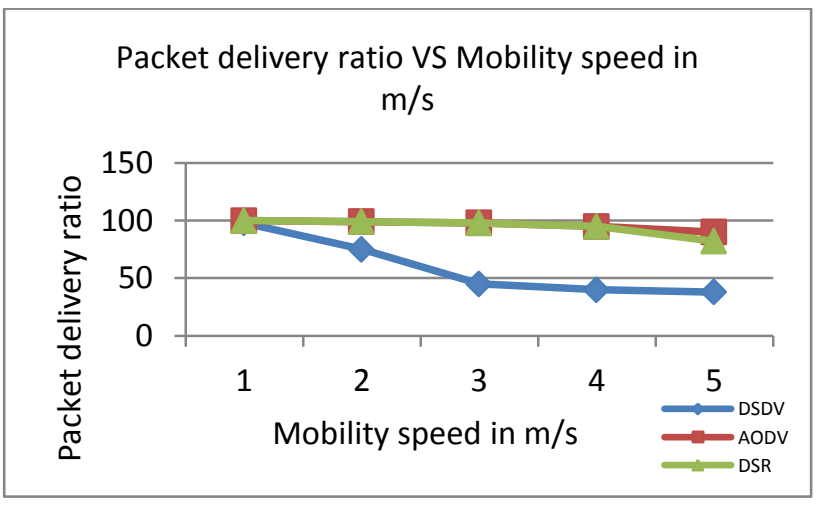

(a) Packet delivery ratio VS Mobility speed

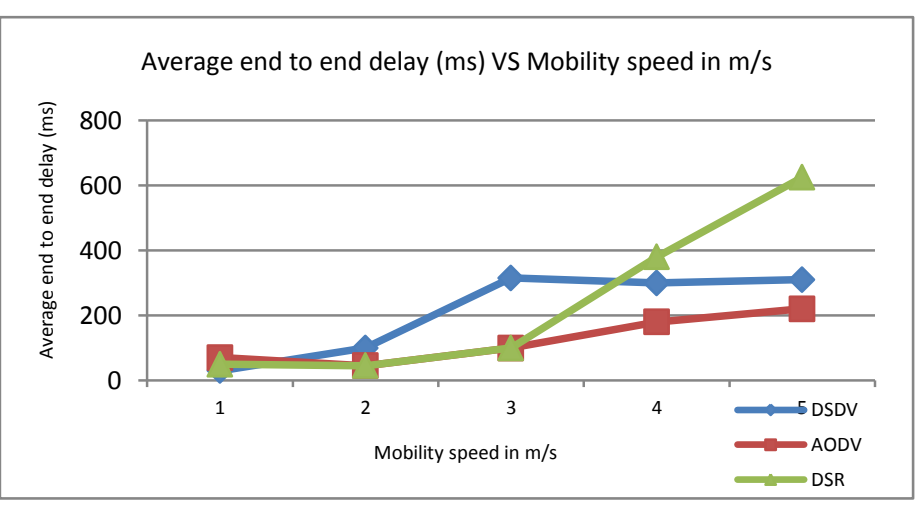

(b) Average end to end delay (ms) VS Mobility speed
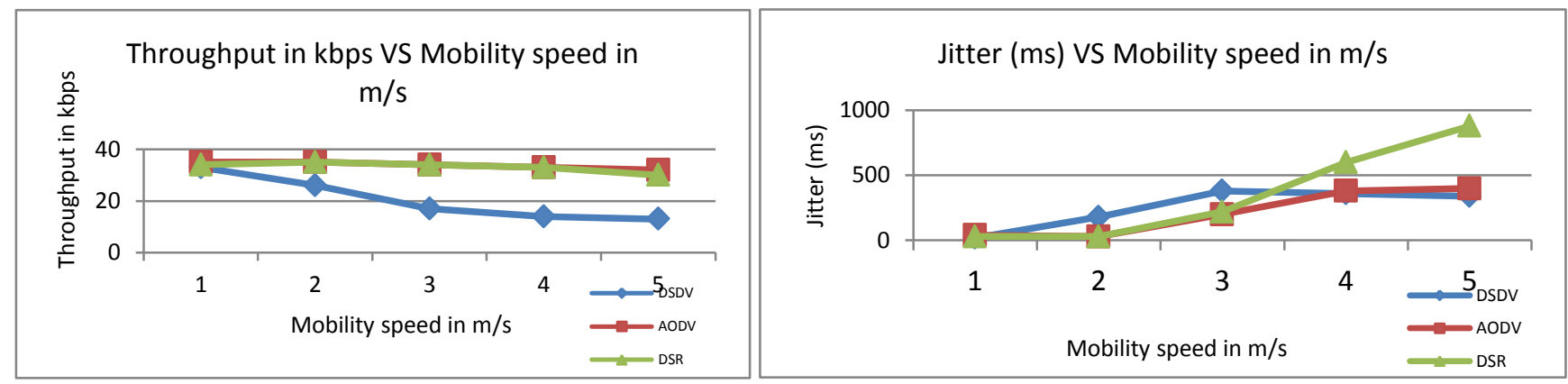
(c) Throughput in kbps VS Mobility speed

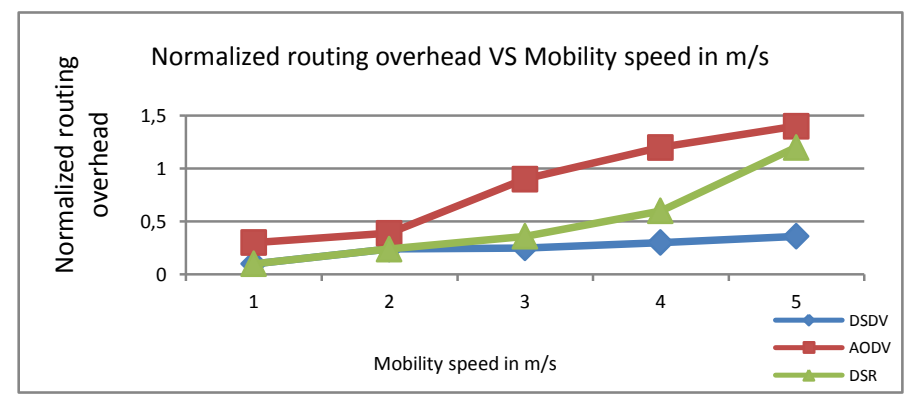

(e) Normalized routing overhead VS Mobility speed (d) Jitter VS Mobility speed

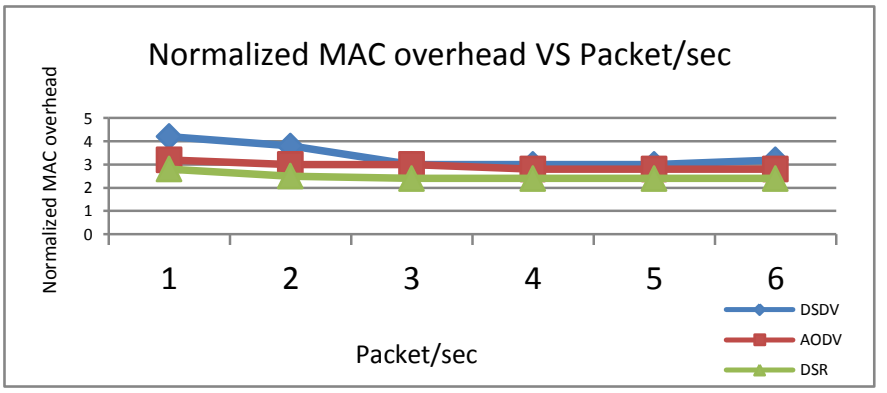

(f) Normalized MAC overhead VS Packet/sec

Figure (2) varying the network size

\subsection{Varying the network size or density in the second set of the simulations:}

The numbers of nodes are varied in the network. The objective is to analyze the impact of node density on the protocol's performance. A desirable property of a protocol is to have stable behavior regardless of the number of nodes in the network. The no. of nodes is gradually increased in the network and takes the values $20,40,60,80$ and 100 . The mobility speed has the range (3-7) $\mathrm{m} / \mathrm{s}$, and the packet rate, no. of connections are fixed at 10 packet/sec and 20 respectively. Other parameters remain fixed for the evaluation of the AODV, DSR and DSDV routing protocols for mobile ad hoc networks.

Packet Delivery Ratio (PDR) in fig (3a), AODV and DSR have almost the same packet delivery ratio while DSDV packet delivery ratio is quite low as compared to reactive protocols because, in DSDV, a node maintains the route for all the nodes available in the network. When the numbers of nodes are increased, DSDV node overhead will also increase. It increases processing delay. Due to this increased processing delay, large no. of data packets are dropped at the node queue. It also happens that DSDV maintains route with more hop count than AODV and DSR. That's why DSDV PDR is deceased when we increase the number of the node from 80 to 100.maintains route with more hop count than AODV and DSR because it maintains route in a proactive manner. That's why DSDV PDR is deceased when we increase the number of the node from 80 to 100. Average End to End Delay In fig (3b), DSDV has a higher end to end delay due to its proactive features. DSR often uses stale routes due to the large route cache, which leads to frequent packet retransmission and high delay times.

AODV uses the source-initiated approach in the route discovery process, and table-driven approach in the route maintenance stage, due to which it shows the better delay characteristics. Throughput Figure (3c) shows that AODV and DSR throughput is almost equal and is better than DSDV because they have higher packet delivery ratio than DSDV. The relatively lower throughput for DSDV is caused by packets that are sent (and lost) before routes have converged initially in the network. Jitter In Fig (3d), AODV shows the smallest rising slope and also displays the most superior jitter performance. Combined with fig 4(a) it can also be seen that its packet delivery ratio is highest which shows that AODV protocol is stable and reliable. 
DSR has higher jitter initially because DSR is the source path routing so finding the path from the source to the destination is more complex and has more overhead in the network (because the size of the control packet is more). Also, the source code requires putting the complete route information in the data packet every time before sending to the destination, so node processing delay is increased which increases the jitter value. But this jitter decreases when the number of nodes increases.

DSDV has more end to end delay than two protocols that's why DSDV has more jitter variation. Normalized Routing Overhead Figure (3e) shows the normalized routing load. DSR has the lowest normalized routing load. Due to aggressive caching, DSR most often finds a route in its cache and therefore rarely initiate a route discovery process, unlike AODV.

But because these routes are most often stale routes, a lot of packets get dropped. DSR's routing overhead is dominated by route replies (unicast packets), while AODV's routing load is dominated by route requests (broadcast packets). Therefore, DSR performs well when looking at the routing overhead. AODV has a higher normalized routing load than DSR and DSDV because of its request broadcasting. When the number of node increases, the request propagation also increases. Also, AODV uses HELLO message to confirm the connectivity of each pair of nodes which leads to bigger overhead than DSDV. Normalized MAC overhead Figure (3f) reveals that DSDV has lowest MAC overhead which shows that it has lower congestion at MAC layer and that too occurs due to control packet generated in the network layer. While in AODV, the MAC overhead is high which reveals that packet is dropped due to contention.

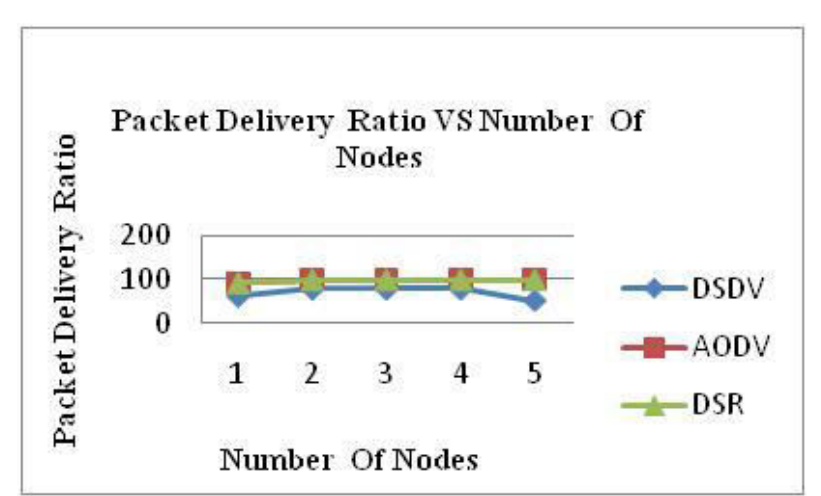

(a) Packet delivery ratio VS number of nodes

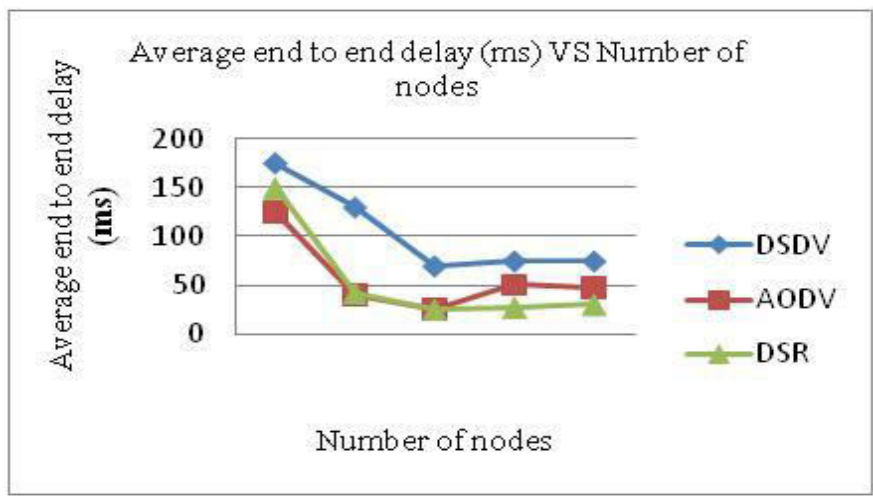

(b)Average end to end delay VS number of nodes

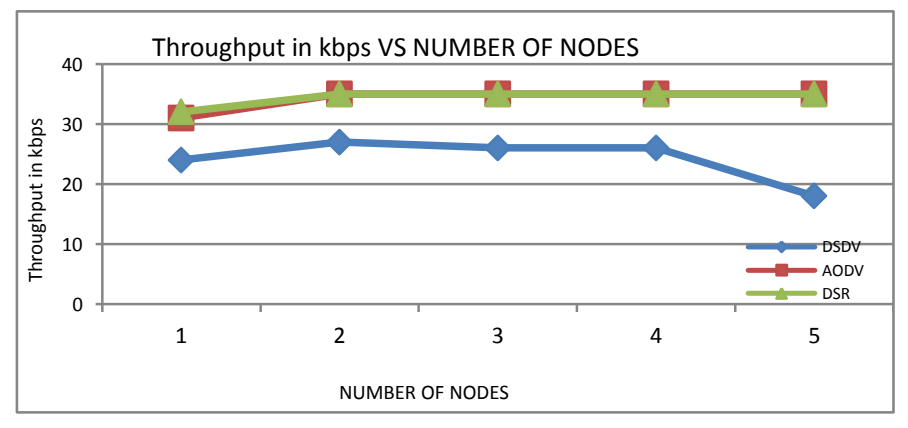

(b) Throughput VS number of nodes

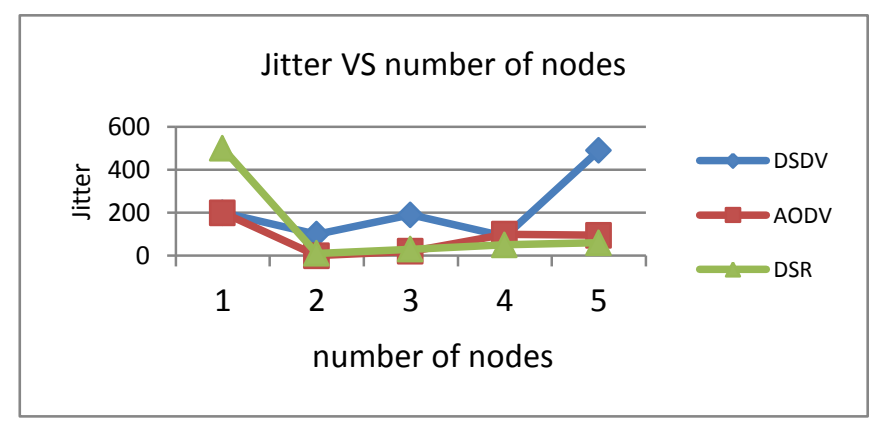

(d) Jitter VS number of nodes 


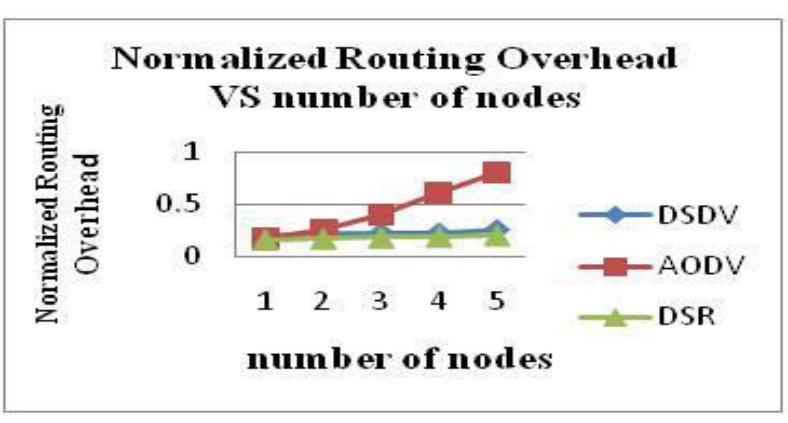

(e) Normalized routing overhead VS number of nodes

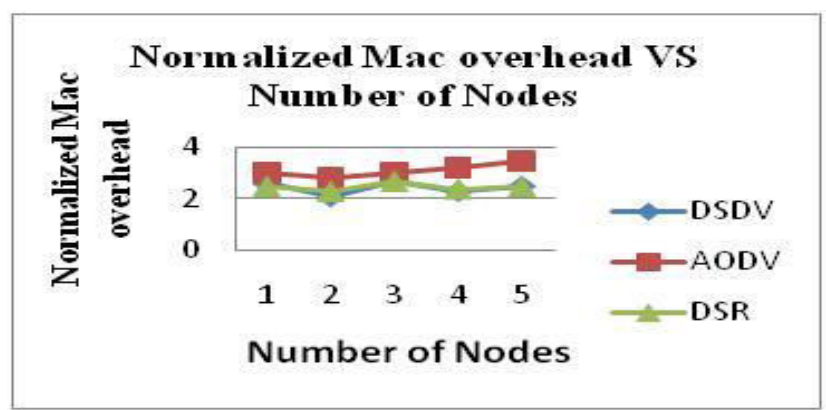

(f) Normalized Mac overhead VS number of nodes

Figure (3) varying the network size

\subsection{Varying the network load in the final set of simulations:}

The number of data packets sent by the sources is increased from 5 packets /s (20.480 kbps) to 20 packets/s $(81.920 \mathrm{kbps})$. The mobility speed has the range $3-7 \mathrm{~m} / \mathrm{s}$, no. of nodes and the connections are fixed at 50 and 20 respectively.

The other parameters remain the same. Packet Delivery ratio Figure (4a) shows the packet delivery ratio of the three protocols where DSR has the highest packet delivery ratio while varying the load. This is because it utilizes the path from route cache and also the processing time is very less than communication time (sends a number of packets with added route information). Above the 12 packet/sec, the packet delivery ratio of all the protocols decreases due to more data load on the network. However, it can be observed that data packets are dropped by AODV for the following reason: in AODV, the source node sends RREQ message only if the route to the destination node does not exist. After the second transmission of the RREQ message, if the source node does not receive an RREP message within the time interval, it will drop the first packet in the queue and repeat the same procedure for the second data packet in the queue.

However, when an intermediate node cannot find a valid route to the destination node by repeating the above procedure, it will drop not only the first packet but also all data packets from its queue, thereby degrading the protocol's performance. Average end to end delay In Fig. (4b), DSDV outperforms DSR and AODV in terms of delay metrics for the increased load in the network.

DSDV is proactive routing protocol so it has a route to the destination all the time. That's why, when the data rate of the node is less, it utilizes the route which is available in the node routing table. When the data rate of the node is increased above 8 packets per second, the CBR packets available in the network are more so congestion occurs which causes the packets to wait in the node for the processing.

This queuing delay ultimately affects the end to end delay of the network that's why a DSDV end to end delay has increased the data rate above 8 packet/s. Reactive routing protocols AODV and DSR have more end to end delay than DSDV. They find the path when the node requires communicating with the destination. Initially, the data rate is less and the control packets are more, so they do not utilize the path efficiently which results in increased delay. 
But when the rate increases, the congestion in the network also increases which results in increased delay. AODV delay is less as compared to DSR because DSR inserts the complete route information in the application packet to the processing delay increases. AODV and DSR performance is almost equal to the data rate of 8 packets/s.

Throughput In fig (4c) AODV and DSR have better throughput than DSDV because these are the reactive protocols and utilize the path information available in the routing table (route cache in case of DSR) and when the data rate increases, no. of CBR packets increases, congestion increases and these reactive routing protocols route to the destination fastly than DSDV protocol.

Jitter In fig (4d), AODV outperforms DSR and DSDV protocol in terms of jitter variation for the increased load in the network because it uses features of both DSDV and DSR routing protocol. DSDV initially has lower jitter than the two protocols because in the network, less no of data packets are available and it utilizes the path information available in the node routing table but when data rate of the node is increased to 12 packets/s or more, congestion increases, number of packets are dropped and thus the jitter increases.

DSDV generates the routing message only after waiting for the hello interval, while in case of reactive protocols, when the network load increases, jitter reduces because they generate the routing messages whenever the route to the destination is invalid (and do not wait for hello interval). DSR has more jitter for the reason described above in case of performance of delay metric.

Normalized Routing Overhead In fig (4e), DSDV has the best performance with an increase of the routing load at a higher mobility. DSR shows lower routing load than AODV as due to aggressive caching, DSR most often finds a route in its cache and therefore rarely initiate a route discovery process, unlike AODV. DSR's routing overhead is dominated by route replies (unicast packets), while AODV's routing load is dominated by route requests (broadcast packets).

Therefore, DSR performs better than AODV in terms of routing overhead. Normalized MAC overhead Figure (4f) shows the normalized MAC load. DSDV again presents the lowest MAC load and AODV has the highest MAC load at lower data packet rates, which drops when the data rates increases.

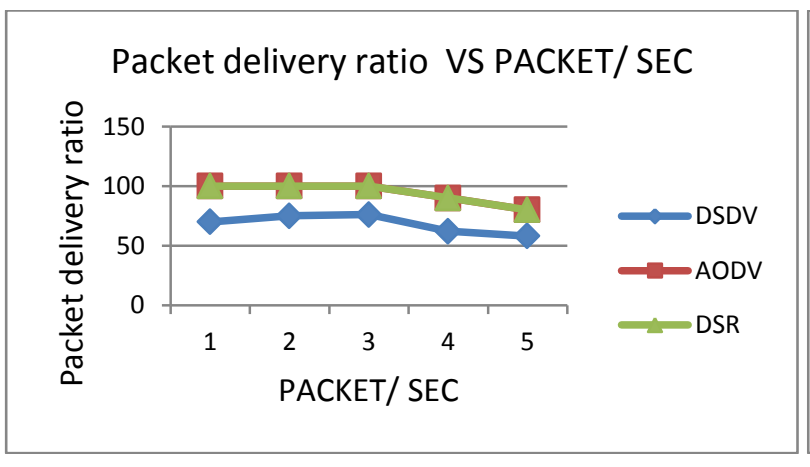

(a) Packet delivery ratio VS Packet / sec

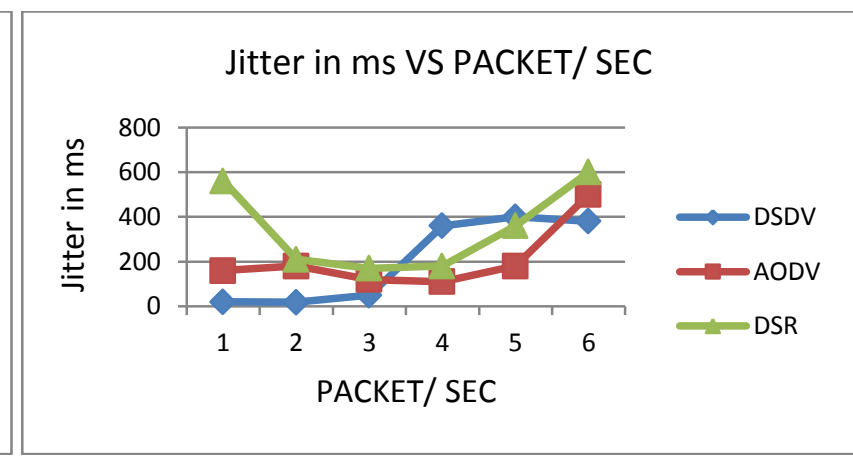

(b) Jitter in ms VS Packet/Sec 


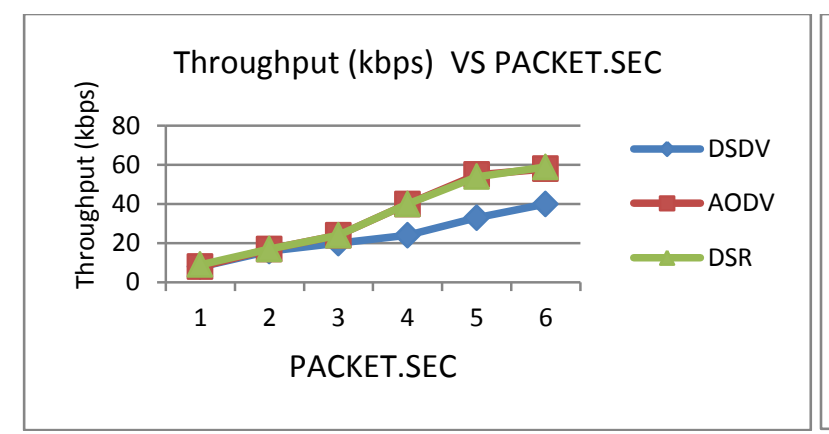

(c) Throughput (kbps) VS Packet /Sec

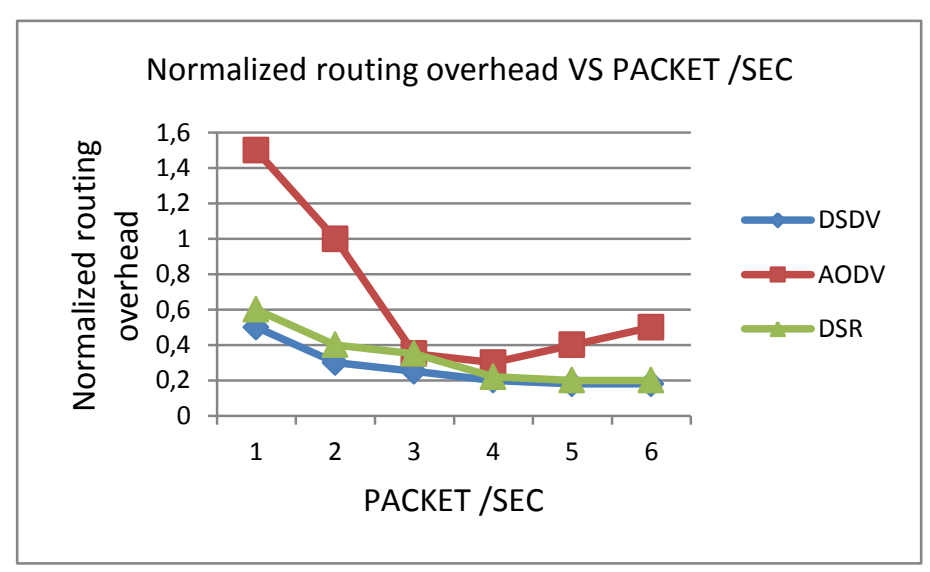

(e) Normalized routing overhead VS Packet /Sec

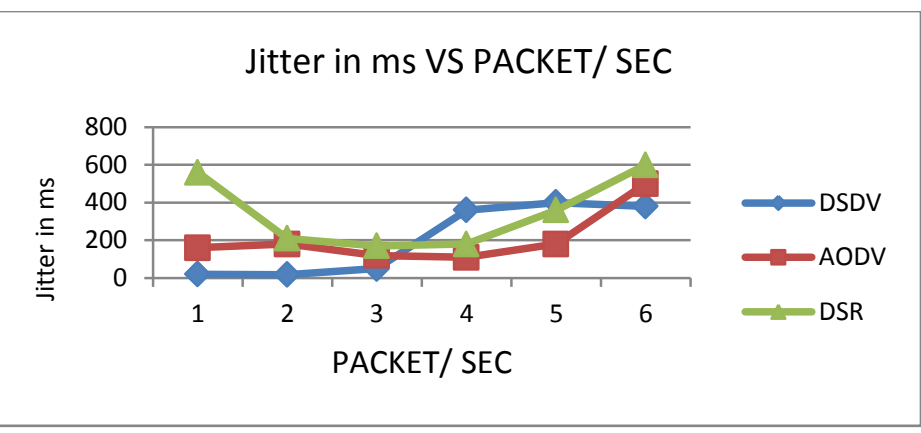

(d) Jitter in ms VS Packet /Sec

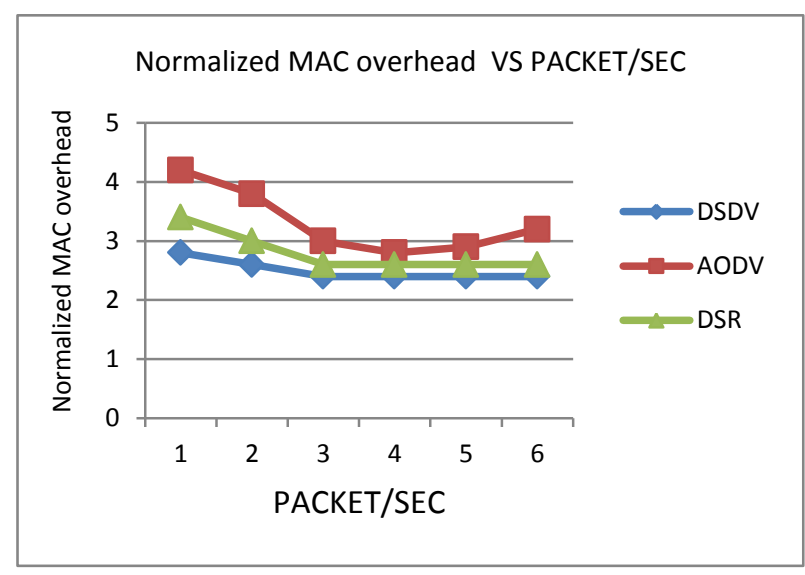

(f) Normalized MAC overhead VS Packet /Sec

Figure (4) varying network load

\section{CONCLUSIONS}

As it can be seen, there is a large number of different kinds of routing protocols in mobile ad-hoc networks, the use of a particular routing protocol in the mobile ad-hoc network depends upon the factors like size of the network, load, mobility requirements etc. This paper compares the performance of DSDV, AODV and DSR routing protocols for mobile ad hoc networks using the NS-2 simulator.

The routing protocols have been compared on the basis of QoS metrics (packet delivery ratio, average end-to-end delay, throughput, jitter, normalized routing load and normalized MAC load by varying mobility speed of the nodes, network size, and the network load. Simulation results show that DSDV is a proactive routing protocol and is suitable for a limited number of nodes with low mobility due to the storage of routing information in the routing table at each node. Since DSR protocol uses source routing and route cache, byte overhead in each packet will increase whenever network topology changes.

Hence, DSR is preferable for moderate traffic with moderate mobility. For the robust scenario where mobility is high, nodes are dense, the amount of traffic is more, AODV performs better among all studied routing protocols. Thus from the simulation results, it can be concluded that for all types of 
networks, AODV performs optimally well not the best.

Though there are some disadvantages of this protocol, it is robust for use in mobile ad hoc networks. Our future work will include the modification to the basic AODV routing protocol so as to make it efficient in providing QoS and meet the challenges of mobile ad hoc networks.

\section{REFERENCES}

1. S. S. Jadhav, A. V. Kulkarni and R. Menon, "Mobile Ad-Hoc Network (MANET) for disaster management," 2014 Eleventh International Conference on Wireless and Optical Communications Networks (WOCN), Vijayawada, 2014, pp. 1-5.

2. A. Sureshkumar, V. Ellappan and K. Manivel, "A comparison analysis of DSDV and AODV routing protocols in mobile AD-HOC networks," 2017 Conference on Emerging Devices and Smart Systems (ICED), Tiruchengode, 2017, pp. 234-237.

3. A. Daas, K. Mofleh, E. Jabr and S. Hamad, "Comparison between AODV and DSDV routing protocols in mobile Ad-hoc Network (MANET)," 2015 5th National Symposium on Information Technology: Towards New Smart World (NSW NSW), Riyadh, 2015, pp. 1-5.

4. M. Manjunath and D. H. Manjaiah, "Spatial DSDV (S-DSDV) routing algorithm for mobile ad hoc network," 2014 International Conference on Contemporary Computing and Informatics (IC3I), Mysore, 2014, pp. 625-629.

5. A. Kaur, "Mobility model-based performance analysis of DSDV mobile ad hoc routing protocol," International Conference on Recent Advances and Innovations in Engineering (ICRAIE-2014), Jaipur, 2014, pp. 1-7.

6. P. Parvathi, "Comparative analysis of CBRP, AODV, DSDV routing protocols in mobile Ad-hoc networks," 2012 International Conference on Computing, Communication and Applications, Dindigul, Tamilnadu, 2012, pp. 1-4.

7. M. M. Morshed, F. I. S. Ko, Dongwook Lim, M. H. Rahman, M. R. Rahman Mazumder and J. Ghosh, "Performance evaluation of DSDV and AODV routing protocols in Mobile Ad-hoc Networks," 4th International Conference on New Trends in Information Science and Service Science, Gyeongju, 2010, pp. 399-403.

8. K. U. R. Khan, R. U. Zaman, A. V. Reddy, K. A. Reddy and T. S. Harsha, "An Efficient DSDV Routing Protocol for Wireless Mobile Ad Hoc Networks and its Performance Comparison," 2008 Second UKSIM European Symposium on Computer Modeling and Simulation, Liverpool, 2008, pp. 506-511.

9. J. Rangaraj and M. Anitha, "Performance analysis of proactive and reactive protocol under different mobility models for MANET," 2017 International Conference on I-SMAC (IoT in Social, Mobile, Analytics, and Cloud) (I-SMAC), Palladam, 2017, pp. 637-643.

10. W. Ullah, H. Ali, A. W. Khan, A. Farhad, B. Ahmad and A. Khan, "Performance assessment of reactive routing protocols in Mobile Ad-hoc Networks under CBR traffic using NS2," 2016 International Conference on Wireless Communications, Signal Processing and Networking (WiSPNET), Chennai, 2016, pp. 1026-1029.

11. A. Sharma and R. Kumar, "Performance comparison and detailed study of AODV, DSDV, DSR, 
TORA and OLSR routing protocols in ad hoc networks," 2016 Fourth International Conference on Parallel, Distributed and Grid Computing (PDGC), Waknaghat, 2016, pp. 732-736.

12. F. F. Khan, T. Samira, A. Jana and K. N. E. A. Siddiquee, "Performance of agro-sensors: Assessment of optimality in routing protocols of MANET in wireless sensor networks," 2016 International Conference on Intelligent Control Power and Instrumentation (ICICPI), Kolkata, 2016, pp. 98-102.

13. Alamsyah, M. H. Purnomo, I. K. E. Purnama and E. Setijadi, "Performance of the routing protocols AODV, DSDV and OLSR in health monitoring using NS3," 2016 International Seminar on Intelligent Technology and Its Applications (ISITIA), Lombok, 2016, pp. 323-328.

14. V. Kumar, A. S. Baghel and P. Mishra, "Performance evaluation of DSDV, AODV, and LSGR protocol in ad-hoc networks," 2016 International Conference on Electrical, Electronics, and Optimization Techniques (ICE OT), Chennai, 2016, pp. 4261-4266.

15. M. N. Aslam, H. A. Alaqel, and S. S. Zaghloul, "A comparative study of MANET routing protocols," The Third International Conference on e-Technologies and Networks for Development (ICeND2014), Beirut, 2014, pp. 178-182.

16. S. Habib, S. Saleem, and K. M. Saqib, "Review on MANET routing protocols and challenges," 2013 IEEE Student Conference on Research and Development, Putrajaya, 2013, pp. 529-533.

17. S. S. Jadhav, A. V. Kulkarni and R. Menon, "Mobile Ad-Hoc Network (MANET) for disaster management," 2014 Eleventh International Conference on Wireless and Optical Communications Networks (WOCN), Vijayawada, 2014, pp. 1-5.

18. M. Kassim, R. Ab. Rahman and R. Mustapha, "Mobile ad hoc network (MANET) routing protocols comparison for a wireless sensor network," 2011 IEEE International Conference on System Engineering and Technology, Shah Alam, 2011, pp. 148-152.

19. M. Patil, R. Naik, B. Nikam, K. Joshi, "Extended ECDSR Protocol for Energy Efficient MANET", International Conference on Advanced Computing and Communication Systems (ICACCS -2015), Jan.05 - 07, 2015.

20. Perkins, C. E., \& Bhagwat, P. (1994, October). Highly dynamic destination-sequenced distancevector routing $(D S D V)$ for mobile computers. In ACM SIGCOMM computer communication review (Vol.24, No. 4, pp. 234-244). ACM.

21. Hasan Abdulwahid, Bin Dai, Benxiong Huang, Zijing Chen, "Scheduled-links multicast routing protocol in MANETs", In Journal of Network and Computer Applications, Volume 63, 2016, Pages 56-67.

22. Zhu Qiankun, Xu Tingxue, Zhou Hongqing, Yang Chunying, Li Tingjun, A Mobile Ad Hoc Networks Algorithm Improved AODV Protocol, In Procedia Engineering, Volume 23, 2011, pp. 229-234.

23. Rendong Bai and M. Singhal, "DOA: DSR over AODV Routing for Mobile Ad Hoc Networks," in IEEE Transactions on Mobile Computing, vol. 5, no. 10, pp. 1403-1416, Oct. 2006.

24. Shihu Xiang, Jun Yang, "Performance reliability evaluation for mobile ad hoc networks", In Reliability Engineering \& System Safety, Volume 169, 2018, pp. 32-39

25. Walikar G. A, Biradar R. C., "Energy-aware multicast routing in mobile ad hoc networks using Ns-2," in Proceedings of the IEEE international conference on Electrical, Computer and Communication Technologies, 2015, pp1536-1542.

26. P.I. Basarkod, S.S. Manvi, Mobility and QoS aware anycast routing in Mobile ad hoc Networks, In Computers \& Electrical Engineering, Volume 48, 2015, pp. 86-99. 